Ärzte müssen auch Zufallsbefunde beachten

Ärzte dürfen „Zufallsbefunde“ nicht außer Acht lassen. Das geht aus einem Urteil des Bundesgerichtshofs hervor. Danach müssen Ärzte auch die Ergebnisse solcher Untersuchungen berücksichtigen, die medizinisch gar nicht indiziert waren. Im Streitfall hatte ein Anästhesist in der Vorbereitung einer Meniskus-Operation die Lunge einer Patientin röntgen lassen. Eine auf ein Karzinom hindeutende Verdichtungszone beachtete der Arzt nicht weiter.

Az.: VI ZR 284/09

Fachgebietsgrenzen sind bindend auch bei Befähigung

Fachärzte bleiben auch dann auf ihr Gebiet beschränkt, wenn sie fachlich in der Lage sind, Leistungen aus anderen Fachgebieten zu erbringen. Das geht aus einer Entscheidung des Hamburgischen Berufsgerichtshofs hervor. Ein Mund-, Kiefer- und Gesichtschirurg, der auch Brustimplantate eingesetzt hatte, muss jetzt wegen Verstoßes gegen das Berufsrecht eine Geldbuße von $1.500 €$ bezahlen. Der Chirurg verwies darauf, dass die Operationen an der Brust weit weniger kompliziert seien als in seinem Fachgebiet. Doch er konnte sich mit seinen Argumenten nicht durchsetzen.

Az.: 6 Bf 60/10.HBG

Hoppe appelliert an Ärzte, seriös zu igeln

Der Präsident der Bundesärztekammer, Professor Jörg-Dietrich Hoppe, hat zum verantwortungsvollen Umgang mit IGeL aufgerufen. Nur wenn Ärzte sich an klare Regeln hielten, könnten sie sich vor Vorwürfen schützen, sie würden Patienten zu Kunden umdefinieren, so Hoppe im „Rheinischen Ärzteblatt“. Patienten müssten darauf vertrauen können, dass medizinische Gründe und nicht das Gewinnstreben Ärzte motivieren, so Hoppe. Dann spreche nichts gegen „ein sachliches und unaufdringliches Gespräch über privat abzurechnende Leistungen". Hoppe reagierte auf Äußerungen der Verbraucherzentrale NRW.

\title{
Urlaubsvertretung als Regressfalle
}

\author{
Spätestens seit einem Urteil des Sozialgerichts Marburg ist klar: Eine \\ Urlaubsvertretung nicht bei der KV anzuzeigen, kann unangenehme \\ Folgen haben.
}

$\mathrm{V}$

iele Vertragsärzte haben die Pflicht, eine Vertretung der KV zu melden, wenn sie länger als eine Woche dauert, bisher häufig vernachlässigt. Der Grund: Es gab letztlich keinen Genehmigungsvorbehalt der Körperschaft, es handelte sich also eigentlich um eine reine Formalie.

Ein aktuelles Urteil des Sozialgerichts Marburg könnte diese Praxis ändern. Denn die Richter begründeten eine Entscheidung über Honorarrückforderungen der KV an eine Praxisgemeinschaft wegen $\mathrm{zu}$ vieler gemeinsamer Patienten auch damit, dass die gegenseitigen Urlaubsvertretungen der beiden Ärzte der KV nicht angezeigt worden waren. Und in einem solchen Fall könne ein Arzt sich nicht auf die Vertretung berufen, so die Richter (S
12 KA 30/2010). „Mit Berufung auf diese Rechtsauffassung könnte eine KV zukünftig die Vergütung der vom Vertreter erbrachten Leistungen verweigern", sagt Rechtsanwalt Dr. Ingo Pflugmacher, jedenfalls dann, wenn sie länger als eine Woche gedauert hat und die KV nicht unverzüglich über die Vertretung informiert worden ist.

Es kann auch andere Anlässe geben, vor einer Praxisvertretung bei der Ärztekammer oder bei der KV nachzufragen, etwa bei Vertretungen in schwerpunktübergreifenden Gemeinschaftspraxen oder dann, wenn der Arzt, der als Vertreter in Frage kommt, noch keine vollwertige Approbation hat. Nur eine vorherige Genehmigung der Körperschaft bringt in solchen Fällen letztlich Rechtssicherheit. ger

\section{Nur echte ärztliche Kooperationen erhalten den RLV-Zuschlag}

Wie bereits erwartet wurde, hat der Bewertungsausschuss die ZuschlagsRegelung für ärztliche Kooperationen angepasst. Entscheidend für die Höhe des Zuschlags zum Regelleistungsvolumen ist demnach der Kooperationsgrad zwischen den Ärzten.

A b dem 3. Quartal dieses Jahres gilt: Nicht mehr die Zahl der Fachgruppen, die an einer Berufsausübungsgemeinschaft (BAG) oder einem MVZ beteiligt sind, ist für das RLV entscheidend, sondern der Kooperationsgrad zwischen den Ärzten. Dieser ergibt sich aus dem Verhältnis der relevanten Arztfälle zu den Behandlungsfällen der Praxis. Das heißt: Je mehr Ärzte einer BAG oder eines MVZ gemeinsam Patienten behandeln, desto höher ist der Kooperationsgrad. Zu beachten ist dabei, dass für das RLV irrelevante Arztfälle - zum Beispiel bei Prävention - nicht angerechnet werden.

Das bedeutet, bei standortübergreifenden fach- und schwerpunktgleichen BAG und Praxen mit angestellten Ärzten der gleichen Arztgruppe beträgt der Zuschlag nach wie vor $10 \%$, wenn auch der Kooperationsgrad mindestens $10 \%$ beträgt. In fach- und schwerpunktübergreifenden BAG und Praxen mit angestellten Ärzte unterschiedlicher Arztgruppen kann der Zuschlag bis zu $40 \%$ bertragen. Liegt der Kooperationsgrad unter $10 \%$, gibt es keinen RLV-Zuschlag. Bei einem Kooperationsgrad zwischen 10 und $15 \%$ beträgt der Zuschlag 10\%. So geht es in FünfProzent-Schritten weiter bis zu einem Zuschlag von maximal $40 \%$.

Für die Berechnung wird das entsprechende Vorjahresquartal herangezogen. Die neue Regelung wird darum vermutlich solchen Zusammenschlüssen zugute kommen, die bereits im vergangenen Jahr Wert auf eine echte Kooperation gelegt haben. 